\title{
Treatment of acute perilunate dislocation or fracture dislocation using dorsal approach and diamond-shaped Kirschner-wire fixation
}

\author{
Tuna Özyürekoğlu, MD ${ }^{1,2} \mathbb{D}$, Mehmet Ali Acar, $M D^{2,3} \mathbb{D}$ \\ ${ }^{1}$ Kleinert Kutz Hand Care Center, Louisville KY, USA. \\ 2Department of Surgery, Division of Hand Surgery, Christine M Kleinert Institute for Hand and Microsurgery, University of Louisville, USA \\ ${ }^{3}$ Department of Orthopedics and Traumatology, Medical School of Selçuk University, Konya, Turkey
}

Perilunate dislocations (PLDs) and perilunate fracture dislocations (PLFDs) are devastating injuries to the wrist. These injuries generally occur in young patients after high-energy trauma ${ }^{[1]}$ The ligamentous and bony structures around the lunate are severely damaged. According to Mayfield et al., ${ }^{[2]}$ the scapholunate (SL), lunocapitate and lunotriquetral (LT) ligaments are sequentially injured, resulting in a lunate dislocation. Fracture dislocations are more common than isolated dislocations. Perilunate fracture dislocations differs from PLD because scaphoid, capitate, triquetrum, radial or ulnar styloid fractures arise at the time of dislocation. ${ }^{[3]}$

The generally accepted treatment for PLDs and PLFDs is an open reduction and internal fixation with a primary repair or ligament reconstruction. ${ }^{[4]}$ The principal factors in successfully treating these

Received: April 28, 2020

Accepted: August 04, 2020

Published online: October 26, 2020

Correspondence: Mehmet Ali Acar, MD. Selçuk Üniversitesi Tıp Fakültesi Ortopedi ve Travmatoloji Anabilim Dalı, 42250 Selçuklu, Konya, Türkiye.

E-mail: dr.mali46@hotmail.com

Doi: $10.5606 /$ ehc. 2021.74838

Citation: Özyürekoğlu T, Acar MA. Treatment of Acute Perilunate Dislocation or Fracture Dislocation using Dorsal Approach and Diamond-Shaped K-Wire Fixation. Jt Dis RelatSurg 2021;32(1):42-50.

(C2021 All right reserved by the Turkish Joint Diseases Foundation

This is an open access article under the terms of the Creative Commons Attribution-NonCommercial License, which permits use, distribution and reproduction in any medium, provided the original work is properly cited and is not used for commercial purposes (http://creativecommons.org/licenses/by-nc/4.0/).

\section{ABSTRACT}

Objectives: This study aims to evaluate the clinical and radiological outcomes of diamond-shaped Kirschner (K)-wire fixation for the treatment of acute perilunate dislocation (PLD) or trans-scaphoid perilunate fracture dislocation (PLFD).

Patients and methods: We performed a retrospective review of 18 patients ( 18 wrists; 17 males, 1 female; mean age $31.5 \pm 2.6$ years; range, 18 to 47 years) treated for PLD/PLFD using a dorsal approach with the diamond-shaped K-wire fixation between November 2001 and September 2017. The mean follow-up of cohort was 27 (range, 13 to 74) months. Using a dorsal approach, open ligament repair and bone fixations were performed. Perilunate dislocation was reduced and the carpal bones and midcarpal joint were held in anatomical position using four K-wires transfixing the scapholunate (SL), lunotriquetral, scaphocapitate, and triquetrohamate joints in such a shape that each bone received two K-wires.

Results: The mean range of motion and grip strength measured using a Jamar ${ }^{\circledast}$ dynamometer of the injured wrist compared to the uninjured extremity were $84.3 \%$ and $78.8 \%$, respectively. The mean Mayo wrist score was 78.3 (range, 70 to 90). The mean Visual Analog Scale score was 1.2 (range, 0 to 4). The average SL gap at the final follow-up evaluation was 1.62 (range, 1 to 2.3) $\mathrm{mm}$. The mean SL and capitolunate angles were $49.3^{\circ}$ (range, 40 to $75^{\circ}$ ) and $4.2^{\circ}$ (range, 2 to $12^{\circ}$ ), respectively.

Conclusion: Because every bone is fixed with two K-wires using this configuration, a closed ring is created; hence no motion is possible between the scaphoid, capitate, hamate, triquetrum, lunate and the midcarpal joint. We believe that diamond-shaped fixation may provide reliable fixation and satisfactory clinical outcomes in patients with PLD and PLFD.

Keywords: Carpal dislocation, K-wire fixation, lunate dislocation, wrist instability, wrist trauma.

dislocations are achieving normal carpal alignment and creating a stable fixation until bone and ligament healing is achieved.$^{[5]}$ Reduction is achieved by surgery for acute PLDs and PLFDs. Maintaining reduction and normal intercarpal relationship is 
the major challenge. ${ }^{[6]}$ Following an open reduction, several temporary or permanent surgical fixation methods are used (Kirschner [K]-wire, screw or cerclage fixation) to maintain immobilization until the ligaments heal. ${ }^{[7]}$ Instability due to inadequate carpal alignment can result in subsequent progressive osteoarthritis, wrist pain and reduced range of movement in the joint. ${ }^{[8]}$ In this study, we aimed to evaluate the clinical and radiological outcomes of diamond-shaped K-wire fixation for the treatment of acute PLD or trans-scaphoid PLFD.

\section{PATIENTS AND METHODS}

This retrospective study was conducted at Christine M Kleinert Institute for Hand and Microsurgery, University of Louisville. Between November 2001 and September 2017, 31 adult patients with acute PLD or PLFD were treated with an open reduction and fixation utilizing a diamond-shaped K-wire and ligament repair. All repairs were performed by the same surgeon.

Among the 31 patients, nine were excluded from the study due to additional injuries, surgical interventions on the same upper extremity, or subacute fixation (more than three weeks). The additional injuries included metacarpophalangeal joint dislocation $(n=1)$, ulnar nerve neuropathy and distal radioulnar joint (DRUJ) dislocation $(n=1)$, radial shaft+radial head fracture+pelvic fracture $(\mathrm{n}=1)$, head and pelvic trauma $(\mathrm{n}=1)$, cervical fracture $(n=1)$, bilateral PLFDs $(n=1)$, and ulnar fracture $(n=1)$. Two patients were excluded due to late fixation older than three weeks. The remaining 22 patients were treated for a unilateral PLD/PLFD with an open reduction with a dorsal approach performed utilizing the diamond-shaped K-wire fixation technique. The medical records and radiographs of the patients were reviewed. An additional four patients were excluded because of follow-up shorter than 12 months or due to insufficient data from their charts. Therefore, 18 patients (18 wrists; 17 males, 1 female; mean age $31.5 \pm 2.6$ years; range, 18 to 47 years) with acute PLD/PLFD were included in the study. The study protocol was approved by the University of Louisville Institutional Review Board Ethics Committee (Date: 2015, No: 14.0539). A written informed consent was obtained from each patient. The study was conducted in accordance with the principles of the Declaration of Helsinki.

The injuries were caused by motor vehicle accidents $(n=6)$, motorcycle accidents $(n=2)$, off-road bike injuries $(n=1)$, all-terrain vehicle injuries $(n=2)$, falling onto the outstretched hand $(n=6)$, and door entrapment $(n=1)$. The right wrist was injured in 10 patients, and the left wrist was injured in eight patients. In eight patients, the dominant wrist was injured. A perilunate injury was diagnosed after standard posteroanterior and lateral plain radiographs of the wrist joint at their initial presentation. The average time between injury and surgery was two (range, 0 to 10) days. The mean follow-up time of cohort was 27 (range, 13 to 74 ) months.

If surgery was scheduled for the same day, the patients were immobilized with a short-arm splint until surgery. If a delay was anticipated, a closed reduction was attempted using a Tavernier's maneuver while the patient was sedated. With axial traction by applying a counter traction maneuver to the capitate at the dislocated midcarpal joint, the wrist was moved from extension to flexion while applying volar pressure to the dislocated lunate. The surgery was performed on 12 patients within 24 hours and five patients within 72 hours. The surgery was performed 10 days after the trauma for one patient.

All surgeries were performed under local anesthesia and using a tourniquet after adequate perioperative antimicrobial prophylaxis. A dorsal approach between Lister's tubercle and the third metacarpal base exposed the wrist joint. The dorsal ligaments and extensor retinaculum were commonly observed stripped from the bone depending on the type and severity of the injury. The carpal bones and torn dorsal ligaments were evaluated. The joint was cleaned of clots, debris, and cartilage pieces. Then, a reduction was attempted. A freer elevator was often placed between the capitate and lunate to facilitate reduction.

For PLDs, a closed reduction was confirmed with mini $\mathrm{C}$-arm guidance. One K-wire was placed in the lunate and another in the scaphoid dorsally as a joystick to manipulate the bones into a normal carpal alignment. Four K-wires were drilled through the radial snuff box and ulnar snuff box in a diamond-shaped pattern: the SL, LT, scaphocapitate, and triquetrohamate joints were transfixed with $1.6 \mathrm{~mm}$ K-wires while maintaining normal carpal angles between the carpal bones and the distal row bones. The injured ligaments were repaired by anchor fixation afterwards. We repaired the SL ligament and the dorsal intercarpal (DIC) ligament using anchors. Using the same anchor and sutures, DIC was repaired as well after the SL reinsertion or repair. For PLFD patients, carpal bone fractures and ligaments were repaired first, because fracture reduction and fixation are possible only before the diamond-shaped pinning. The carpal bones were 


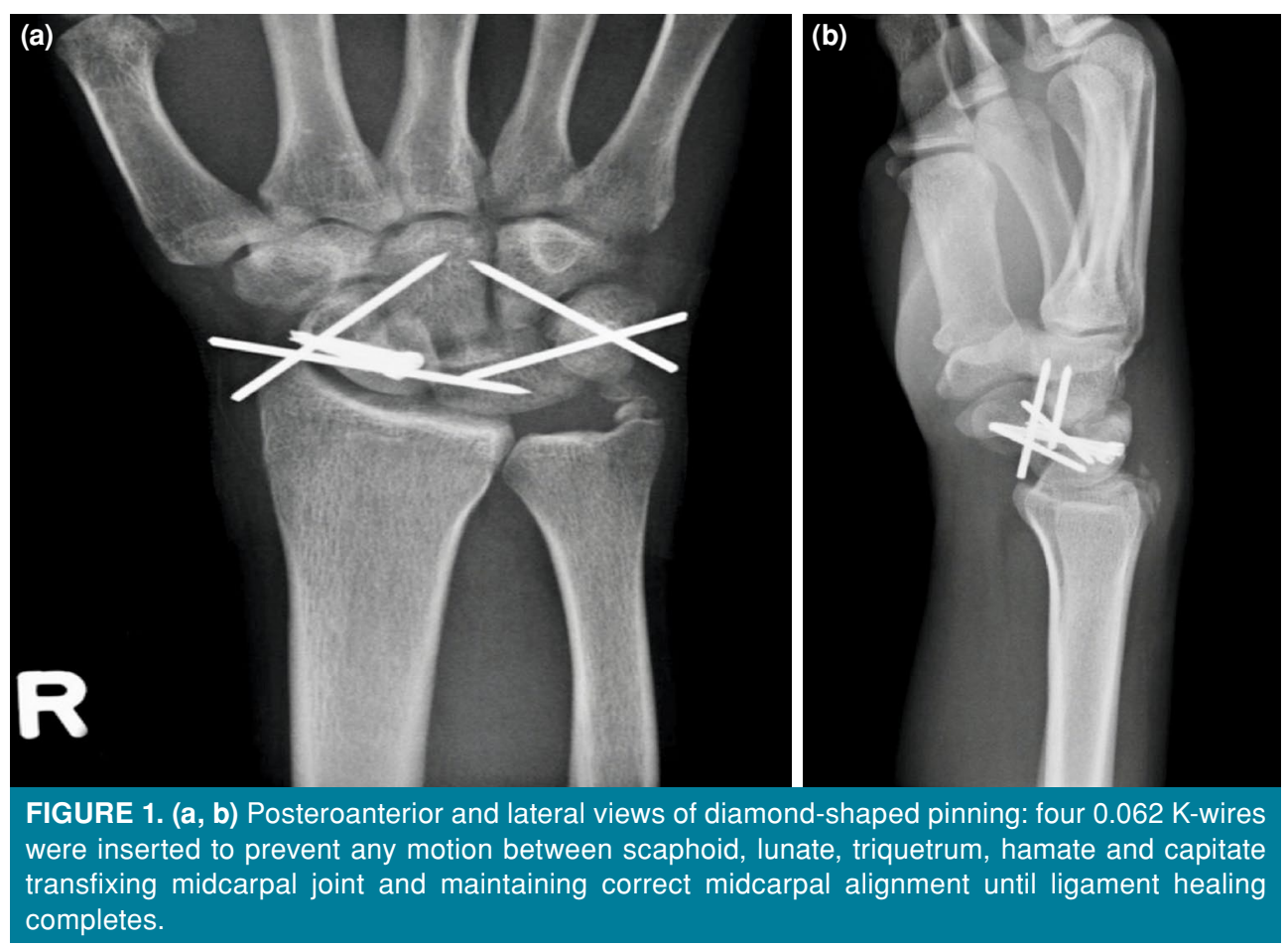

brought into a normal carpal relation, and then the $\mathrm{K}$-wires were passed. DIC ligament was repaired if found avulsed. Then, four K-wires were passed in a diamond-shaped configuration. The ends of the
K-wires were buried under the skin (Figure 1a, b). We also reinserted the ulnar attachment of the distal radioulnar ligaments through fixation of the ulnar styloid when the DRUJ was found unstable.

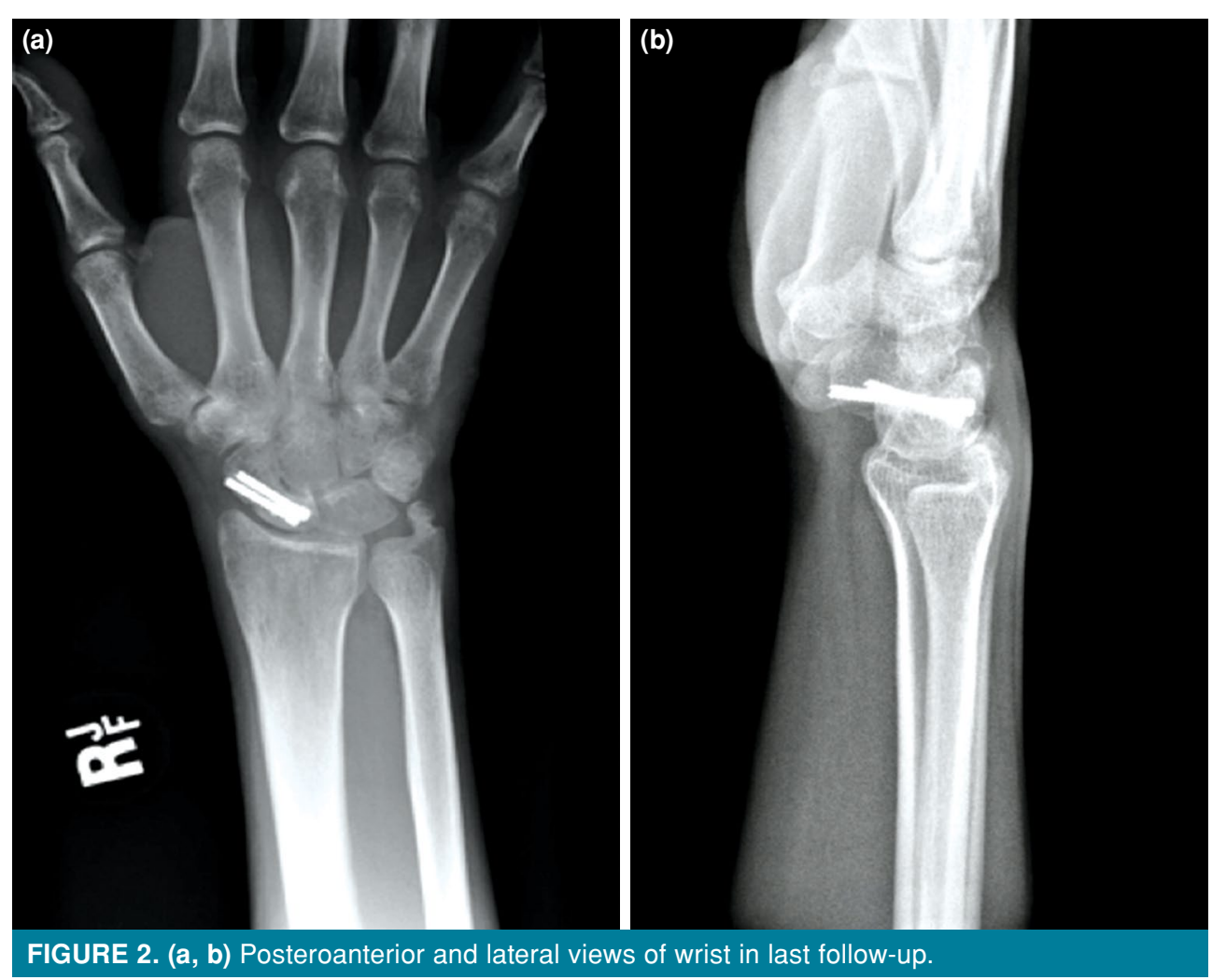




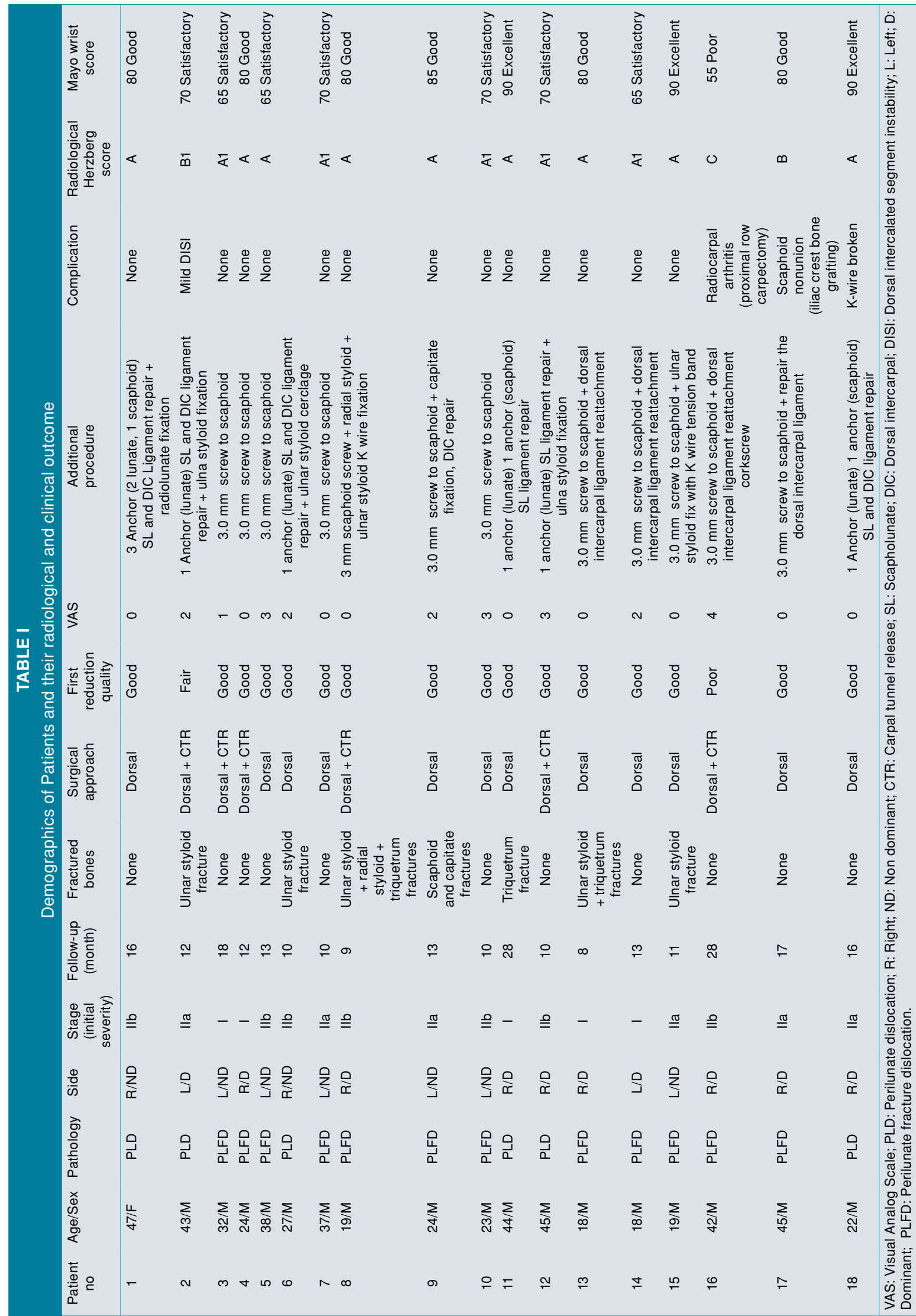


For patients with a concomitant ulnar styloid fracture and DRUJ instability, a long-arm splint was applied with the forearm in a neutral position. For patients with an intact ulnar styloid, a short-arm splint was applied, and the arm was immobilized for eight weeks. Following eight weeks, the K-wires were removed under local anesthesia, and the patient was referred to physical therapy for range of motion exercises (Figure $2 \mathrm{a}, \mathrm{b}$ ).

Clinical data included age, sex, hand dominance, mechanism of injury, time from injury to surgery and length of follow-up. The severity of the initial injury was classified according to the Radiological Herzberg Score. The range of motion was measured with a hand-held goniometer, and grip strength was measured with a Jamar ${ }^{\circledR}$ Hydraulic Hand Dynamometer (Sammons Preston, Bolingbrook, Illinois, USA). A functional assessment was performed by an independent reviewer using the Mayo Wrist Score and Visual Analog Scale score. The radiological outcomes included a Herzberg classification, SL gap at the time of surgery and at the final follow-up examination, SL, capitolunate (CL) angle, dorsal intercalated segment instability (DISI), and presence or absence of midcarpal arthritis (Table I).

\section{Statistical analysis}

In this retrospective study database was analyzed using Microsoft excel. Charts were also created using Microsoft excel. At baseline and the follow-up assessment, mean values and standard deviation of data collected from clinical and subjective measures were calculated.

\section{RESULTS}

Among the 18 patients included in this study, adequate scaphoid and intercarpal bone alignment was obtained and maintained in all patients treated for both PLDs and PLFDs. Among the six patients treated for PLDs, the Mayo Wrist Scores were excellent in two patients, good in one patient and satisfactory in three patients. The mean Mayo Wrist Score was 78.3 (range, 70 to 90). No permanent pain was reported by any of the patients. One patient reported occasional pain. One patient experienced occasional pain during daily activities. One patient experienced occasional pain during heavy manual work. At the final follow-up, these three patients reported no pain. Compared with the uninjured side, the mean wrist movement (flexion-extension)

\begin{tabular}{|c|c|c|c|c|c|c|}
\hline \multicolumn{7}{|c|}{$\begin{array}{c}\text { TABLE II } \\
\text { gth in patients }\end{array}$} \\
\hline Patient no & $\begin{array}{l}\text { Extension-flexion } \\
\text { arc }\end{array}$ & $\begin{array}{l}\text { Extension-flexion } \\
\text { arc (contralateral) }\end{array}$ & $\begin{array}{l}\text { Extension-flexion } \\
\text { (\% contralateral) }\end{array}$ & $\begin{array}{c}\text { Grip strength } \\
(\mathrm{kg})\end{array}$ & $\begin{array}{l}\text { Grip strength } \\
\text { (contralateral) }\end{array}$ & $\begin{array}{c}\text { Grip } \\
(\% \text { contralateral })\end{array}$ \\
\hline 1 & 100 & 120 & 0.83 & 30 & 40 & 0.75 \\
\hline 2 & 80 & 125 & 0.64 & 30 & 50 & 0.60 \\
\hline 3 & 115 & 130 & 0.88 & 40 & 50 & 0.80 \\
\hline 4 & 95 & 120 & 0.79 & 35 & 40 & 0.88 \\
\hline 5 & 105 & 140 & 0.75 & 35 & 45 & 0.78 \\
\hline 6 & 110 & 145 & 0.76 & 45 & 50 & 0.90 \\
\hline 7 & 120 & 130 & 0.92 & 35 & 45 & 0.78 \\
\hline 8 & 140 & 145 & 0.97 & 40 & 50 & 0.80 \\
\hline 9 & 130 & 130 & 1.00 & 40 & 50 & 0.80 \\
\hline 10 & 120 & 140 & 0.86 & 35 & 40 & 0.88 \\
\hline 11 & 130 & 150 & 0.87 & 45 & 55 & 0.82 \\
\hline 12 & 110 & 120 & 0.92 & 30 & 35 & 0.86 \\
\hline 13 & 130 & 145 & 0.90 & 40 & 50 & 0.80 \\
\hline 14 & 95 & 125 & 0.76 & 45 & 60 & 0.75 \\
\hline 15 & 120 & 130 & 0.92 & 50 & 50 & 1.00 \\
\hline 16 & 80 & 130 & 0.62 & 20 & 45 & 0.44 \\
\hline 17 & 105 & 140 & 0.75 & 30 & 40 & 0.75 \\
\hline 18 & 115 & 130 & 0.88 & 55 & 55 & 1.00 \\
\hline
\end{tabular}




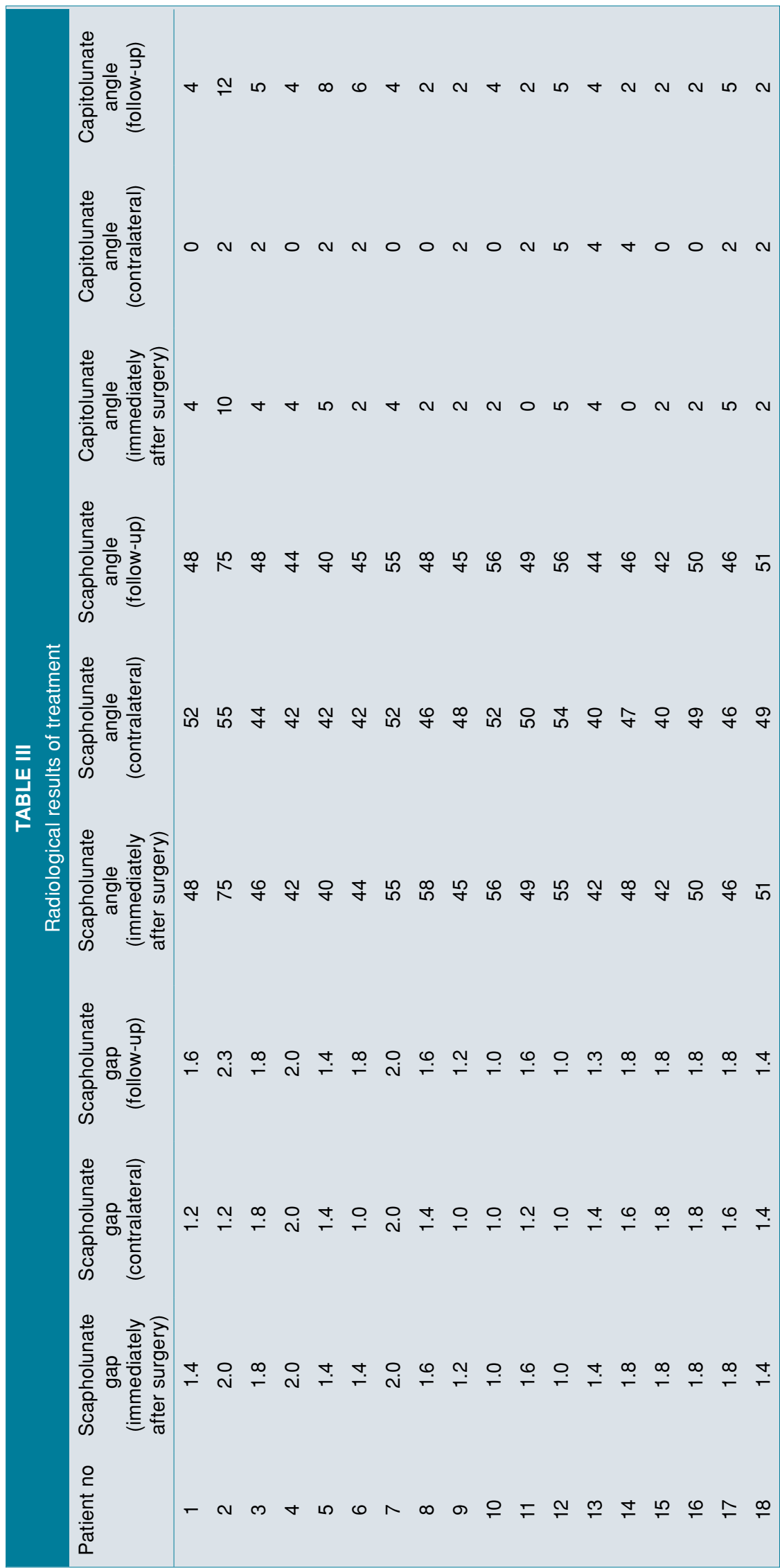


and grip strength (Jamar ${ }^{\circledR}$ dynamometer) rates were $81.7 \%$ and $82.1 \%$, respectively. The mean active flexion-extension arc was $108^{\circ}$ (range, 80 to $130^{\circ}$ ).

In the PLFD group, the Mayo Wrist Score was excellent in one patient, good in six patients, satisfactory in four patients and poor in one patient. The mean Mayo Wrist Score was 74.6 (range, 55 to 90). At the final follow-up, six patients reported no pain. Two patients reported occasional pain. One patient reported occasional pain during daily activities. Three patients reported occasional pain during heavy manual work. No continuous pain was reported by any of the patients. The mean active flexionextension arc was $113^{\circ}$ (range, 80 to $140^{\circ}$ ) (Table II). The mean wrist motion in flexion-extension arc and grip strength (Jamar ${ }^{\circledR}$ dynamometer) were $84.3 \%$ and $78.8 \%$ of the uninjured side, respectively.

The radiological evaluations are summarized in Table III. At the final follow-up, the evaluations according to the Herzberg classification were three Type A cases, two Type A1 cases and one Type B1 case in the PLD group and seven Type A cases, three Type A1 cases, one Type B case and one Type C case (proximal row carpectomy) in the PLFD group. At the final follow-up evaluation, the average SL gap was 1.6 (range, 1 to 2.3 ) $\mathrm{mm}$. The mean SL and CL angles were $49.3^{\circ}$ (range, 40 to $75^{\circ}$ ) and $4.2^{\circ}$ (range, 2 to $12^{\circ}$ ), respectively.

Posttraumatic arthritic changes were observed in seven patients involving the radioscaphoid joint in five patients (mostly sclerosis and osteophytes), the lunocapitate joint in one patient and at both joints in one patient. In one patient, there was an osteochondral fragment on the lateral surface of the scaphoid. We applied it as a graft. The triquetral fractures consisted of osteochondral fragments; however, as they were reduced well after the reduction of the LT joint, we did not attempt to approach these volarly. Carpal bone fractures healed well with pin fixation. In one patient, there was a chondral fragment in the scaphoid but this was reduced with the SL ligament repair with anchors. As for complications: in the PLFD group, a scaphoid nonunion developed in one patient. Following an iliac crest bone graft and repeat fixation, union was achieved. Avascular necrosis did not develop in our series. In one patient who presented only after three months postoperatively, one K-wire was found broken at the SL joint due to shearing motion at the joint. Despite the broken K-wire, the function of the wrist was not affected. Another patient in the PLFD group had poor scaphoid fracture reduction and fixation. He later underwent a proximal row carpectomy.

\section{DISCUSSION}

Perilunate dislocations and PLFDs are rare injuries and constitute approximately $3 \%$ of all carpal injuries. PLFD injuries are 50\% more likely than isolated PLD injuries. ${ }^{[9]}$ Mayfield et al. ${ }^{[2]}$ studied the carpal kinematics of progressive perilunate instability and emphasized the key role of ligamentous injuries. Certainly, early surgical treatment is required for these injuries. When diagnosis and treatment are delayed, patients experience poor outcomes, including restricted wrist movements, pain and osteoarthritis. ${ }^{[2]}$

A closed reduction is currently the first step in treating PLDs. General carpal alignment is provided by a closed reduction and can increase patients` level of comfort. However, closed reduction does not sufficiently restore the anatomy and provide a reliable carpal relationship. Therefore, in patients with PLDs and PLFDs, it is important to achieve normal carpal alignment with surgical methods and to protect this alignment with fixation methods until healing occurs.

Several authors reported dorsal, volar or combinations of these surgical approaches..$^{[10-12]} \mathrm{A}$ combined approach provides good visualization, facilitates the anatomic reduction, and allows the transverse carpal ligament and median nerve to be released ${ }^{[9]}$ With the volar approach, the volar capsular ligaments can be repaired. In contrast, the combined approach has disadvantages of postoperative adhesions, avascular necrosis and tightness in the wrist due to the wide dissection. ${ }^{[13]} \mathrm{In}$ the current study, only a dorsal approach was utilized in all 18 patients. The dorsal ligaments were repaired directly and the thick ligaments on the volar side were reduced indirectly and held in place by K-wires. Once the lunate was reduced, compression on the median nerve was relieved; however, because of the bleeding into the carpal tunnel or due to continued symptoms of median nerve despite reduction, we released the carpal tunnel through a mini incision in six $(33 \%)$ patients in the series.

Different fixation methods have been reported to maintain the reduction achieved during PLD treatment. ${ }^{[7,14-16]}$ These methods include temporary headless compression screws on the SL and LT joint, lag screws, cerclage wires or K-wires. Diamondshaped pinning maintained the reduction between the scaphoid and lunate, lunate and triquetrum and kept the proximal row against the distal row in a normal anatomical alignment ${ }^{[16]}$ Because every bone is fixed with two K-wires using this configuration, a closed ring is created; hence no motion is possible between the scaphoid, capitate, hamate, triquetrum 
and lunate. Our results showed that the reduction was maintained in all patients not only in terms of SL or LT gapping but also in terms of DISI or volar intercalated segment instability deformity. ${ }^{[17]}$ Effective pinning until recovery of the ligaments seems to be the key factor for long-term clinical success of PLD and PLFD treatments.

Trumble and Verheyden ${ }^{[9]}$ reported SL and LT ligament repair using an interosseous cerclage wire in isolated PLDs. In their technique, the LT ligament anchor and LT were fixed with K-wires, and fixation of the SL joint was achieved with a cerclage wire. During this fixation method with a combined approach, the cerclage wire broke in 14 patients. The broken wires required removal of the implants and reoperations. ${ }^{[9]}$ In a study by Budoff, ${ }^{[18]}$ a SL lag screw or headless compression screw (a more rigid fixation method) was used as a temporary fixation. With this method, better compression was claimed compared to the K-wires. The disadvantages of the K-wires were listed as pin tract infections, tendon irritation and the loss of early movement. Both fixation methods performed by Budoff ${ }^{[18]}$ and Trumble and Verheyden, ${ }^{[9]}$ fixed only the proximal row. In both studies, the interosseous cerclage or lag screw could close the gap between the two carpal bones but there was no fixation to resist the extension or flexion of the proximal carpal row.

Currently, the most applied fixation method is fixation with temporary K-wires. ${ }^{[10,14]}$ Although K-wires were used by many authors, no specified fixation recommendation exists in the literature. The K-wire configurations were mostly arbitrary and could mainly be observed in the figures published. In a study by Hildebrand et al., ${ }^{[19]}$ PLD and PLFD treatments were performed using a fixation method with two K-wires for both the SL and LT fixed to only the proximal row. In another study by Sotereanos et al., ${ }^{[13]}$ the same fixation method was applied. However, they only used one K-wire. Herzberg ${ }^{[10]}$ and Malović et al. ${ }^{[20]}$ reported successful results from fixation to the proximal row and the midcarpal joint with a K-wire. The pinning technique described in this article follows a biomechanical reasoning that delivers stability not only by the position of each bone relative to each other, but also by the position of the proximal row as a whole against the distal carpal row. Our results of four K-wires in a diamond-shaped configuration for the treatment of PLD or PLFD showed that effective fixation is achieved by two non-parallel K-wires passing through each bone segment and between the proximal and distal rows. ${ }^{[21]}$

This study had a few limitations including its retrospective design and lack of comparing the outcomes of different techniques. We also did not evaluate the long-term degenerative changes in the wrist. Further prospective and comparative studies with longer follow-up times and larger patient groups are needed to determine whether our technique is superior to other fixation techniques.

In conclusion, in this relatively large and homogenous cohort of patients with PLD and PLFD, we believe that diamond-shaped fixation may provide reliable fixation and satisfactory clinical outcomes. Since every bone fixation is supplied via two K-wires in a closering configuration, no motion is possible betwen the proximal and distal carpal row.

\section{Declaration of conflicting interests}

The authors declared no conflicts of interest with respect to the authorship and/or publication of this article.

\section{Funding}

The authors received no financial support for the research and/or authorship of this article.

\section{REFERENCES}

1. Martinage A, Balaguer T, Chignon-Sicard B, Monteil MC, Dréant N, Lebreton E. Perilunate dislocations and fracturedislocations of the wrist, a review of 14 cases. Chir Main 2008;27:31-9.

2. Mayfield JK, Johnson RP, Kilcoyne RK. Carpal dislocations: pathomechanics and progressive perilunar instability. J Hand Surg Am 1980;5:226-41.

3. Garg B, Goyal T, Kotwal PP. Staged reduction of neglected transscaphoid perilunate fracture dislocation: a report of 16 cases. J Orthop Surg Res 2012;7:19.

4. Weil WM, Slade JF 3rd, Trumble TE. Open and arthroscopic treatment of perilunate injuries. Clin Orthop Relat Res 2006;445:120-32.

5. Herzberg G, Forissier D. Acute dorsal trans-scaphoid perilunate fracture-dislocations: medium-term results. J Hand Surg Br 2002;27:498-502.

6. Apergis E, Maris J, Theodoratos G, Pavlakis D, Antoniou N. Perilunate dislocations and fracture-dislocations. Closed and early open reduction compared in 28 cases. Acta Orthop Scand Suppl 1997;275:55-9.

7. Souer JS, Rutgers M, Andermahr J, Jupiter JB, Ring D. Perilunate fracture-dislocations of the wrist: comparison of temporary screw versus K-wire fixation. J Hand Surg Am 2007;32:318-25.

8. Cooney WP, Bussey R, Dobyns JH, Linscheid RL. Difficult wrist fractures. Perilunate fracture-dislocations of the wrist. Clin Orthop Relat Res 1987;(214):136-47.

9. Trumble T, Verheyden J. Treatment of isolated perilunate and lunate dislocations with combined dorsal and volar approach and intraosseous cerclage wire. J Hand Surg Am 2004;29:412-7.

10. Herzberg G. Perilunate and axial carpal dislocations and fracture-dislocations. J Hand Surg Am 2008;33:1659-68.

11. Kremer T, Wendt M, Riedel K, Sauerbier M, Germann G, Bickert B. Open reduction for perilunate injuries-clinical outcome and patient satisfaction. J Hand Surg Am 2010;35:1599-606. 
12. Dunn JC, Koehler LR, Kusnezov NA, Polfer E, Orr JD, PirelaCruz MA, et al. Perilunate dislocations and perilunate fracture dislocations in the U.S. Military. J Wrist Surg 2018;7:57-65.

13. Sotereanos DG, Mitsionis GJ, Giannakopoulos PN, Tomaino $\mathrm{MM}$, Herndon JH. Perilunate dislocation and fracture dislocation: a critical analysis of the volar-dorsal approach. J Hand Surg Am 1997;22:49-56.

14. Chou YC, Hsu YH, Cheng CY, Wu CC. Percutaneous screw and axial Kirschner wire fixation for acute transscaphoid perilunate fracture dislocation. J Hand Surg Am 2012;37:715-20.

15. Osman K, Momoh P, Ruiz A. An extreme case of transcaphoid-transcapitate perilunate dislocation in an adolescent. J Hand Surg Eur Vol 2015;40:862-3.

16. Bhatia DN. Arthroscopic reduction and stabilization of chronic perilunate wrist dislocations. Arthrosc Tech 2016;5:e281-90.

17. Atik OŞ. What are the expectations of an editor from a scientific article? Jt Dis Relat Surg 2020;31:597-8.

18. Budoff JE. Treatment of acute lunate and perilunate dislocations. J Hand Surg Am 2008;33:1424-32.

19. Hildebrand KA, Ross DC, Patterson SD, Roth JH, MacDermid JC, King GJ. Dorsal perilunate dislocations and fracture-dislocations: questionnaire, clinical, and radiographic evaluation. J Hand Surg Am 2000;25:1069-79.

20. Malović M, Pavić R, Milosević M. Treatment of transscaphoid perilunate dislocations using a volar approach with scaphoid osteosynthesis and temporary Kirschner wire fixation. Mil Med 2011;176:1077-82.

21. Atik OŞ. Is there something new and interesting in my article? Eklem Hastalik Cerrahisi 2019;30:69. 